\title{
Communication
}

\section{Phenotype of the Aging-Dependent Spontaneous Onset of Hearing Loss in DBA/2 Mice}

\author{
Min-Soo Seo ${ }^{1,+}{ }^{,}$Byeonghyeon Lee ${ }^{2,3,{ }^{\dagger}}$, Kyung-Ku Kang ${ }^{1}$, Soo-Eun Sung ${ }^{1}{ }^{\circledR}$, Joo-Hee Choi ${ }^{1}$, Si-Joon Lee ${ }^{1}$, \\ Young-In Kim ${ }^{4}$, Young-Suk Jung ${ }^{5}$, Un-Kyung Kim ${ }^{2,6, *, \ddagger}$ and Kil Soo Kim ${ }^{1,7, *, \neq}$ \\ 1 Laboratory Animal Center, Daegu-Gyeongbuk Medical Innovation Foundation, Daegu 41016, Korea; \\ msseo@dgmif.re.kr (M.-S.S.); kangkk@dgmif.re.kr (K.-K.K.); sesung@dgmif.re.kr (S.-E.S.); \\ cjh522@dgmif.re.kr (J.-H.C.); sjlee1013@dgmif.re.kr (S.-J.L.) \\ 2 Department of Biology, College of Natural Sciences, Kyungpook National University, Daegu 41016, Korea; \\ lbh2080@knu.ac.kr \\ 3 Advanced Bio-Resource Research Center, Kyungpook National University, Daegu 41566, Korea \\ 4 KPC Corporation, Gwangju 12773, Korea; kimyoungin@kpclab.co.kr \\ 5 College of Pharmacy, Pusan National University, Busan 46241, Korea; youngjung@pusan.ac.kr \\ 6 KNU Creative BioResearch Group (BK21 Plus Project), School of Life Sciences, Kyungpook National \\ University, Daegu 41016, Korea \\ 7 Department of Veterinary Toxicology, College of Veterinary Medicine, Kyungpook National University, \\ Daegu 41566, Korea \\ * Correspondence: kimuk@knu.ac.kr (U.-K.K.); kskim728@knu.ac.kr (K.S.K.); \\ Tel.: +82-53-950-5353 (U.-K.K.); +82-53-950-7792 (K.S.K.); \\ Fax: +82-52-950-3066 (U.-K.K.); +82-52-950-5955 (K.S.K.) \\ + Seo and Lee contributed equally to this work as co-first authors. \\ $\ddagger \quad$ Kim and Kim contributed equally to this work as co-corresponding authors.
}

Citation: Seo, M.-S.; Lee, B.; Kang, K.-K.; Sung, S.-E.; Choi, J.-H.; Lee, S.-J.; Kim, Y.-I.; Jung, Y.-S.; Kim, U.-K.; Kim, K.S. Phenotype of the Aging-Dependent Spontaneous Onset of Hearing Loss in DBA/2 Mice. Vet. Sci. 2021, 8, 49. https://doi.org/ $10.3390 /$ vetsci8030049

Academic Editor: Ellen (Liz) Sparger

Received: 12 January 2021

Accepted: 15 March 2021

Published: 17 March 2021

Publisher's Note: MDPI stays neutral with regard to jurisdictional claims in published maps and institutional affiliations.

Copyright: (c) 2021 by the authors. Licensee MDPI, Basel, Switzerland. This article is an open access article distributed under the terms and conditions of the Creative Commons Attribution (CC BY) license (https:// creativecommons.org/licenses/by/ $4.0 /)$.
Simple Summary: In this study, we confirmed the changes in hearing function and inner ear structure over a long period of time in DBA/2 mice, a laboratory animal model suitable for studying hearing loss. We believe that our study is the first to report findings regarding hearing function and structural changes in DBA/ 2 mice aged $\geq 34$ weeks. These results are of significance for researchers and the scientific community using laboratory animal models.

Abstract: DBA/2 mice are a well-known animal model for hearing loss developed due to intrinsic properties of these animals. However, results on the phenotype of hearing loss in DBA $/ 2$ mice have been mainly reported at an early stage in mice aged $\leq 7$ weeks. Instead, the present study evaluated the hearing ability at 5,13 , and 34 weeks of age using DBA/2korl mice. Auditory brainstem response test was performed at $8-32 \mathrm{KHz}$ at 5,13 , and 34 weeks of age, and hearing loss was confirmed to be induced in a time-dependent manner. In addition, histopathological evaluation at the same age confirmed the morphological damage of the cochlea. The findings presented herein are the results of the long-term observation of the phenotype of hearing loss in DBA/ 2 mice and can be useful in studies related to aging-dependent hearing loss.

Keywords: hearing loss; laboratory animals; aging; DBA/2 mice

\section{Introduction}

With the development of modern civilization, individuals are at an increased risk of exposure to excessive noise. Moreover, with the increase in the number of aging individuals, senile hearing loss is emerging as a major social and environmental concern [1]. As reported by the World Health Organization, hearing loss has increased worldwide, with 360 million individuals currently suffering from hearing loss [2]. It is known that senile hearing loss increases gradually at first but then increases rapidly with age [3].

Studies using laboratory animals have great merits in that the research environment can be controlled [4]. In addition to supplying drinking water and food; preventing 
exposure to the external environment; and controlling light, temperature, humidity, and noise; the age of the laboratory animal can be selected according to the study situation [5]. In this case, animal ages for examination of hearing functions were selected to reflect changes in hearing typical of senile hearing loss [6-8].

In this study, DBA/2 mice, which are frequently used in hearing-loss-related research studies [6,9], were used to measure the changes in hearing threshold with increasing age using the auditory brainstem response $(\mathrm{ABR})$ test to evaluate the structural changes in the cochlea by histopathological analysis. Through these approaches, we aimed to obtain basic data for hearing loss research in DBA/2 mice.

\section{Materials and Methods}

\subsection{Animals}

Male DBA/2korl mice were kindly provided by the Department of Laboratory Animal Resources at the National Institute of Food and Drug Safety Evaluation (NIFDS, Cheongju, Korea). The mice had access to a standard irradiated chow diet (Purina, Seoul, Korea) and sterilized water ad libitum. In this study, the animal facility conditions were maintained under a strict light cycle (light on-off system, $12 \mathrm{~h}$ dark-light cycle, 07:00-19:00), $23 \pm 2{ }^{\circ} \mathrm{C}$, and $50 \pm 10 \%$ relative humidity under specific pathogen-free conditions. To evaluate hearing loss in the DBA/2korl mice, we used 37 male mice for ABR test and 15 male mice for histopathological analysis. ABR was recorded and histopathological analysis was conducted at 5, 13, and 34 weeks of age. The animal experimental protocols were reviewed and approved by the Institutional Animal Care and Use Committee of The Laboratory Animal Center of the DGMIF (IACUC; approval No. DGMIF-20011601-01) and were in accordance with their guidelines.

\subsection{ABR Test}

Auditory functions were assessed by recording ABRs using an ABR workstation (System 3; Tucker Davis Technology (TDT), Inc., Alachua, FL, USA), as described previously [10]. All tests were conducted in a soundproof room. Briefly, the mice were anesthetized by an intramuscular injection of a mixture of tiletamine-zolazepam $(1.8 \mathrm{mg} / 100 \mathrm{~g})$ and xylazine hydrochloride $(0.7 \mathrm{mg} / 100 \mathrm{~g})$. The mice were then placed on a heating pad to maintain their body temperature at $37^{\circ} \mathrm{C}$. The body temperature of the mice was monitored using a rectal thermometer. ABRs were recorded by inserting subcutaneous needle electrodes into the vertex (+charge), mastoid ( $-\mathrm{e})$, and hind leg (ground). Acoustic stimuli, which included a tone-burst stimulus with a $1 \mathrm{~ms}$ rise/fall time and a $5 \mathrm{~ms}$ plateau at frequencies of 8,16 , and $32 \mathrm{kHz}$ or transient click stimuli, were monaurally applied using a speaker. Stimulus signals were generated using SigGenRP and an RP2.1 real-time processor and were transmitted using a programmable attenuator (PA5 and TDT), a speaker driver (ED1 and TDT), and an electrostatic speaker (MF1 and TDT). The stimuli were generated for 500 repetitions at $5 \mathrm{~dB}$ decrements, starting from a sound pressure level of $90 \mathrm{~dB}$ to the acoustic threshold at each frequency. The phase of the stimulus was reversed after each presentation to decrease the artifacts caused by repetitive stimuli.

\subsection{Histological Analysis}

After recording the ABRs, the mice were perfused with $4 \%$ paraformaldehyde (PFA) in phosphate-buffered saline (PBS) and their inner ears were isolated. For preparing paraffin sections, the inner ears were fixed with $4 \%$ PFA in PBS for $24 \mathrm{~h}$ at $4{ }^{\circ} \mathrm{C}$ and then decalcified with $10 \%$ ethylenediaminetetraacetic acid in PBS for $24 \mathrm{~h}$ at $4{ }^{\circ} \mathrm{C}$. The specimens were dehydrated using a graded ethanol series, permeabilized with xylene, and embedded in paraffin. The paraffin-embedded inner ears were serially sectioned to obtain 6 - $\mu \mathrm{m}$-thick sections using a microtome (Leica RM2235; Leica Microsystems, Nussloch, Germany), mounted on Superfrost Plus microscope slides (Fisher Scientific, Pittsburgh, PA, USA), and then subjected to H\&E staining. 


\subsection{Statistical Analysis}

The results were analyzed using Student's $t$-test and Excel (Microsoft, Redmond, WA, USA) and are presented as mean \pm standard error in graphical plots. Statistically significant differences are indicated using asterisks. $p$ values of $<0.0001$ were considered statistically significant.

\section{Results}

\subsection{Measurement of Auditory Function with Increasing Age}

In the present study, four stimuli (transient click and 8, 16, and $32 \mathrm{kHz}$ ) were delivered at 5,13 , and 34 weeks of age in the mice, according to the average hearing thresholds (Figure 1A-D). At 5 weeks of age, a hearing threshold value of $\geq 40 \mathrm{~dB}$ was confirmed at a high-frequency stimulation of $32 \mathrm{kHz}$. From 13 weeks of age, the average hearing threshold value appeared to increase rapidly, thereby causing hearing loss. In particular, a hearing threshold value was recorded of $\geq 70 \mathrm{~dB}$ in transient click and $8 \mathrm{kHz}$ stimuli and $\geq 80 \mathrm{~dB}$ in 16 and $32 \mathrm{kHz}$ stimuli in a 34-week-old elderly mouse. These results indicate that significant hearing loss was developed at an early stage in the DBA/2korl mice, especially at high frequencies (Figure 1A-D). In addition, it was confirmed that changes in the hearing threshold were markedly increased in an aging-dependent manner.

A

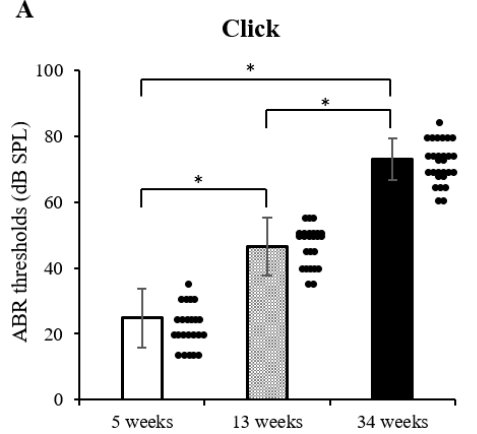

C

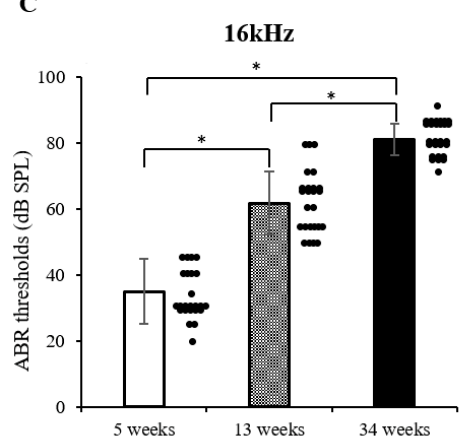

B

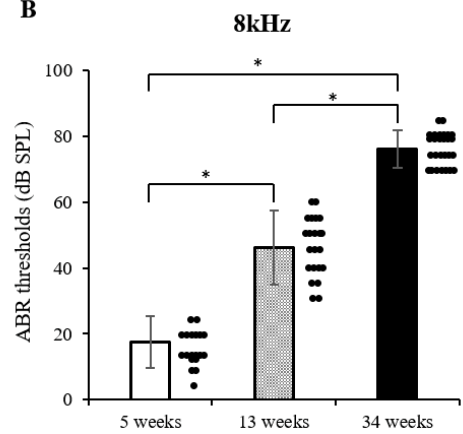

D

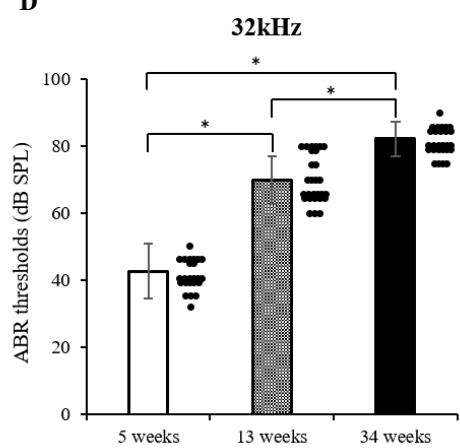

Figure 1. Patterns of hearing threshold in DBA/2korl mice, as revealed by the auditory brainstem response (ABR test): (A) transient click, (B) $8 \mathrm{kHz},(\mathbf{C}) 16 \mathrm{kHz}$, and (D) $32 \mathrm{kHz}$ stimuli. Data are presented as mean \pm standard error. * Significantly different compared with each group $(n=37$, $* p<0.0001)$. The data are shown as the mean \pm SE.

\subsection{Histopathological Analysis of Cochlea with Increasing Age}

To observe the structural changes in the cochlea of the inner ear with increasing age in DBA/2korl mice, a histopathological analysis was performed using H\&E staining at 5,13 , and 34 weeks of age (Figure $2 \mathrm{~A}-\mathrm{C}$ ). The structures of the apex, middle, and basal parts of the cochlea were confirmed. At 5 weeks of age, a normal histopathological structure was observed (Figure 2A). At 13 weeks of age, degeneration of the hair cells in the middle turn and basal turn of the organ of Corti was observed (Figure 2B), which became particularly pronounced at 34 weeks of age, when overall degeneration was observed. 
Furthermore, it was confirmed that the spiral ganglion exhibited degeneration at 34 weeks of age (Figure $2 \mathrm{C}$ ). These results suggest that hearing loss is associated with damage to the structure of the cochlea.
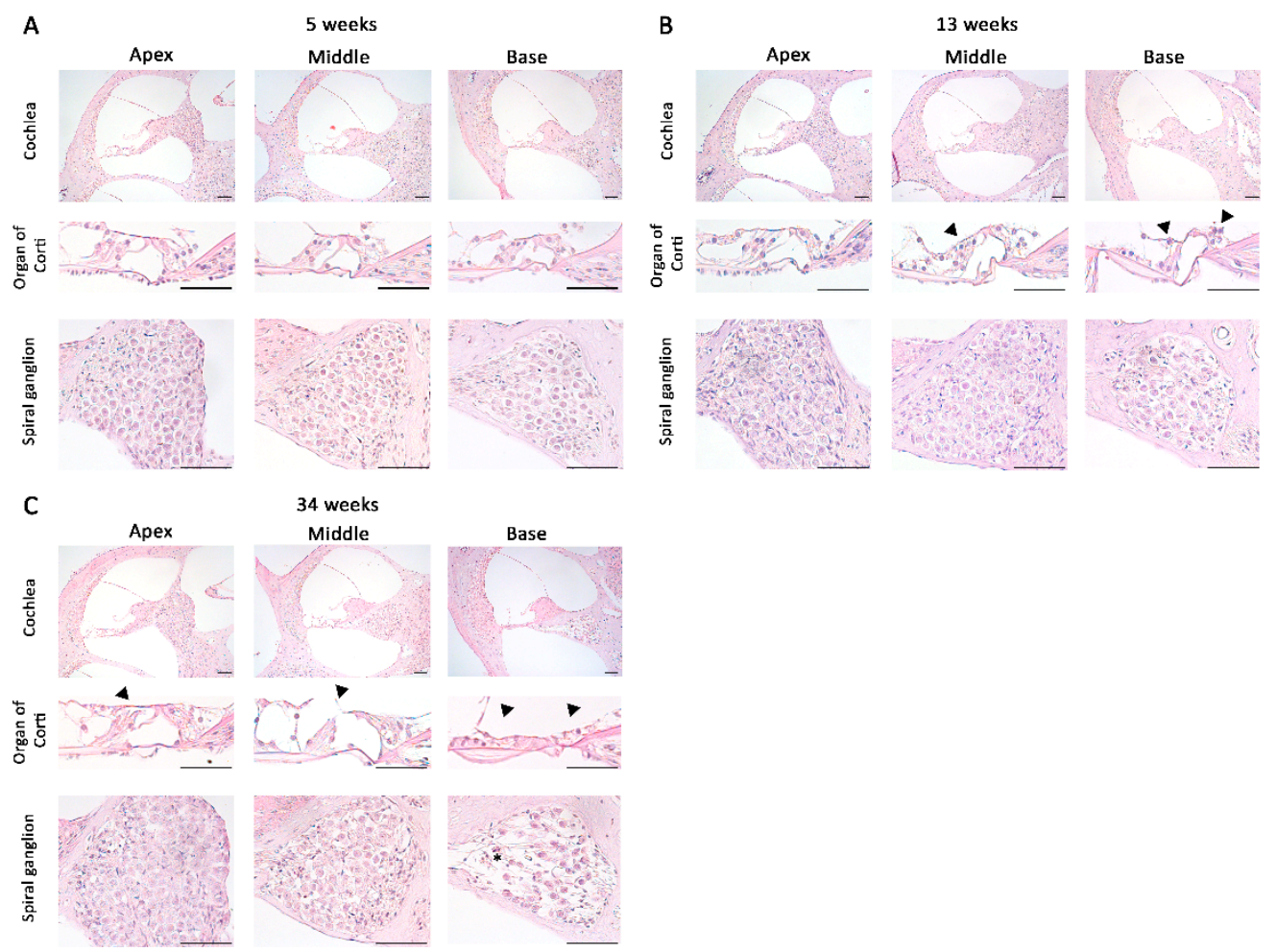

Figure 2. Histopathological analysis of the cochlea in DBA/2korl mice: images of the cochlea of DBA/2 mice at (A) 5, (B) 13, and (C) 34 weeks of age. Black arrowhead: degenerative site on the organ of Corti; asterisk: degenerative site on the spiral ganglion. Scale bars, $100 \mu \mathrm{m}$.

\section{Conclusions}

DBA/2 mice, an inbred mouse strain, have been widely used as disease models for research related to hearing loss and neuronal degeneration $[9,11]$. DBA/2 mice have three recessive alleles focused on $\mathrm{Cdh} 23 \mathrm{ahl}$, which can induce progressive cochlear damage affecting the inner ear's organ of Corti [12]. DBA/2 mice have normal hearing function at 2-3 weeks of age, but then they gradually develop hearing loss in the high-frequency region. It has been reported that at 5 weeks of age, the hearing threshold increases by $30 \mathrm{~dB}$ at $\geq 8 \mathrm{kHz}$ and severe hearing loss occurs at 2-3 months of age [13]. However, to the best of our knowledge, hearing function and histopathological findings have not been reported in DBA/ 2 mice aged $\geq 24$ weeks to date. The ABR test is the primary noninvasive method used to evaluate hearing function, and its findings have been previously reported in laboratory animals [14-16]. Therefore, the aim of our study was to investigate hearing function and histopathological findings in elderly DBA/2 mice.

In the present study, the ABR test revealed that the hearing thresholds shifted to higher frequencies in elderly DBA/2korl mice. In the organ of Corti in the cochlea, damage such as hair cell loss was observed from 13 weeks of age. These hair cells are extremely fragile and cannot regenerate after being damaged [17]. In the case of spiral ganglion, degeneration was observed at 34 weeks of age.

Our study confirmed that this change in hearing function is senile hearing loss, which occurs rapidly in the high-frequency region with age. Taken together, the results obtained here should be useful for senile deafness-related research, understanding the phenotype of laboratory animals, and evaluating the efficacy of new drugs. Of the several mouse models associated with the age-related hearing loss mouse model, the most widely used mouse 
strain is probably C57BL/6. However, as shown in the results of the previous study [18], C57BL/ 6 showed age-related hearing loss gradually from 3-5 months, whereas in DBA/2 mice, ABR thresholds are changed more than $20 \mathrm{~dB}$ starting from 2 months. Thus, there are more meaningful results about early progressive hearing loss. Although our results focus on the ABR test and histopathology, we tried to compare it sequentially from the younger weeks of 5 weeks old to the older weeks of 13 and 34 weeks of age. These results can be used as reference data for the 5 weeks and 34 weeks of age in the hearing loss study using DBA/2 mice. In addition, DBA/2 mice have shown a tendency to induce auditory seizures in previous studies [19-21], and it is known that the $c d h 23^{a h l}$ allele causes age-related hearing loss [12]. These findings suggest that the genetic background of DBA2 mice influences the result of hearing loss with age. Therefore, the DBA2 mouse can be a suitable mouse model for studying the connection between hearing and seizures and the mechanism of hearing loss.

Author Contributions: K.S.K. and U.-K.K. designed and supervised the entire study. K.-K.K., S.-E.S., J.-H.C., S.-J.L. and Y.-I.K. conducted the laboratory animal management and study. B.L. contributed to evaluation of hearing function. Y.-S.J. reviewed the data and manuscript. M.-S.S. analyzed the data and wrote the manuscript. All authors have read and agreed to the published version of the manuscript.

Funding: This study was supported by a grant from National Laboratory Animal Resources (NLAR) from the Ministry of Food and Drug Safety in 2018.

Institutional Review Board Statement: This study was approved by the Institutional Animal Care and Use Committee of The Laboratory Animal Center of the Daegu-Gyeongbuk Medical Innovation Foundation (IACUC; approval No. DGMIF-20011601-01) and were in accordance with the guidelines of the "Guide for the Care and Use of Laboratory Animals" of Daegu-Gyeongbuk Medical Innovation Foundation.

Informed Consent Statement: Not applicable.

Data Availability Statement: The data presented in this study are available on reasonable request from the corresponding author.

Conflicts of Interest: The authors declare that they have no conflict of interest in this paper.

\section{References}

1. Davis, A.; McMahon, C.M.; Pichora-Fuller, K.M.; Russ, S.; Lin, F.; Olusanya, B.O.; Chadha, S.; Tremblay, K.L. Aging and Hearing Health: The Life-course Approach. Gerontology 2016, 56 (Suppl. 2), S256-S267. [CrossRef]

2. Horvath, L.; Bachinger, D.; Honegger, T.; Bodmer, D.; Naldi, A.M. Functional and morphological analysis of different aminoglycoside treatment regimens inducing hearing loss in mice. Exp. Ther. Med. 2019, 18, 1123-1130. [CrossRef]

3. Rigters, S.C.; Van Der Schroeff, M.P.; Papageorgiou, G.; Baatenburg, d.J.R.J; Goedegebure, A. Progression of Hearing Loss in the Aging Population: Repeated Auditory Measurements in the Rotterdam Study. Audiol. Neurotol. 2018, 23, 290-297. [CrossRef] [PubMed]

4. Yamauchi, C. Studies on the Environmental Control of Laboratory Animals. Exp. Anim. 1995, 44, 9-21. [CrossRef]

5. Nevalainen, T. Animal Husbandry and Experimental Design. ILAR J. 2014, 55, 392-398. [CrossRef] [PubMed]

6. Turner, J.G.; Parrish, J.L.; Hughes, L.F.; Toth, L.A.; Caspary, D.M. Hearing in laboratory animals: Strain differences and nonauditory effects of noise. Comp. Med. 2005, 55, 12-23. [PubMed]

7. Gi, M.; Shim, D.B.; Wu, L.; Bok, J.; Song, M.H.; Choi, J.Y. Progressive hearing loss in vitamin A-deficient mice which may be protected by the activation of cochlear melanocyte. Sci. Rep. 2018, 8, 16415. [CrossRef]

8. Yang, L.; Zhang, H.; Han, X.; Zhao, X.; Hu, F.; Li, P.; Xie, G.; Gao, L.; Cheng, L.; Song, X.; et al. Attenuation of hearing loss in DBA/2J mice by anti-apoptotic treatment. Hear. Res. 2015, 327, 109-116. [CrossRef] [PubMed]

9. Kondo, T.; Saigo, S.; Ugawa, S.; Kato, M.; Yoshikawa, Y.; Miyoshi, N.; Tanabe, K. Prebiotic effect of fructo-oligosaccharides on the inner ear of DBA/2 J mice with early-onset progressive hearing loss. J. Nutr. Biochem. 2020, 75, 108247. [CrossRef]

10. Manji, S.S.; Miller, K.A.; Williams, L.H.; Dahl, H.-H.M. Identification of Three Novel Hearing Loss Mouse Strains with Mutations in the Tmc1 Gene. Am. J. Pathol. 2012, 180, 1560-1569. [CrossRef] [PubMed]

11. McLin, J.P.; Steward, O. Comparison of seizure phenotype and neurodegeneration induced by systemic kainic acid in inbred, outbred, and hybrid mouse strains. Eur. J. Neurosci. 2006, 24, 2191-2202. [CrossRef]

12. Johnson, K.R.; Longo-Guess, C.; Gagnon, L.H.; Yu, H.; Zheng, Q.Y. A locus on distal chromosome 11 (ahl8) and its interaction with Cdh23ahl underlie the early onset, age-related hearing loss of DBA/2J mice. Genomics 2008, 92, 219-225. [CrossRef] [PubMed] 
13. Ahn, J.H.; Kang, H.H.; Kim, T.Y.; Shin, J.-E.; Chung, J.W. Lipoic acid rescues DBA mice from early-onset age-related hearing impairment. NeuroReport 2008, 19, 1265-1269. [CrossRef]

14. Ingham, N.J.; Pearson, S.; Steel, K.P. Using the Auditory Brainstem Response (ABR) to Determine Sensitivity of Hearing in Mutant Mice. Curr. Protoc. Mouse Biol. 2011, 1, 279-287. [CrossRef]

15. Dong, Y.; Guo, C.; Chen, D.; Chen, S.; Peng, Y.; Song, H.; Shi, J. Association between age-related hearing loss and cognitive decline in C57BL/6J mice. Mol. Med. Rep. 2018, 18, 1726-1732. [CrossRef] [PubMed]

16. Honkura, Y.; Suzuki, J.; Sakayori, N.; Inada, H.; Kawase, T.; Katori, Y.; Osumi, N. Effects of enriched endogenous omega-3 fatty acids on age-related hearing loss in mice. BMC Res. Notes 2019, 12, 1-7. [CrossRef]

17. Ison, J.R.; Allen, P.D.; O’Neill, W.E. Age-Related Hearing Loss in C57BL/6J Mice has both Frequency-Specific and Non-FrequencySpecific Components that Produce a Hyperacusis-Like Exaggeration of the Acoustic Startle Reflex. J. Assoc. Res. Otolaryngol. 2007, 8, 539-550. [CrossRef] [PubMed]

18. Smith, M.E.; Groves, A.K.; Coffin, A.B. Editorial: Sensory Hair Cell Death and Regeneration. Front. Cell. Neurosci. 2016, 10, 208. [CrossRef]

19. Chapman, A.G.; Croucher, M.J.; Meldrum, B.S. Evaluation of anticonvulsant drugs in DBA/2 mice with sound-induced seizures. Arzneimittelforschung 1984, 34, 1261-1264.

20. Chapman, A.G.; De Sarro, G.M.; Premachandra, M.; Meldrum, B.S. Bidirectional effects of beta-carbolines in reflex epilepsy. Brain Res Bull. 1987, 19, 337-346. [CrossRef]

21. Engstrom, F.L.; Woodbury, D.M. Seizure Susceptibility in DBA and C57 Mice: The Effects of Various Convulsants. Epilepsia 1988, 29, 389-395. [CrossRef] [PubMed] 